\title{
Differential Expression of MicroRNAs Between 21A Genetic Male Sterile Line and Its Maintainer Line in Cotton (Gossypium hirsutum L.)
}

\author{
Yujuan Zhang ${ }^{1}$, Zujun Yin ${ }^{2}$, Xuemei Feng ${ }^{1} \&$ Fafu Shen ${ }^{1}$ \\ ${ }^{1}$ State Key Laboratory of Crop Biology, College of Agronomy, Shandong Agricultural University, Tai'an 271018, \\ Shandong, People's Republic of China \\ ${ }^{2}$ State Key Laboratory of Cotton Biology, Cotton Research Institute, Chinese Academy of Agricultural Sciences, \\ Anyang 455100, Henan, People's Republic of China \\ Correspondence: Fafu Shen, State Key Laboratory of Crop Biology, College of Agronomy, Shandong \\ Agricultural University, Tai'an 271018, Shandong, People's Republic of China. Tel: 86-538-824-2903. E-mail: \\ ffshen@sdau.edu.cn
}

Received: July 17, 2013 Accepted: September 24, 2013 Online Published: September 27, 2013

doi:10.5539/jps.v3n1p13 URL: http://dx.doi.org/10.5539/jps.v3n1p13

\begin{abstract}
MicroRNAs (miRNAs) are a family of 19- to 25-nucleotides small non-coding RNAs that play important roles in regulation of many developmental processes in animals and plants, including flower development. In this study, the cotton (Gossypium hirsutum L.) genetic male sterile (GMS) line and its maintainer line were used to detect miRNAs related to male sterility. Using a bioinformatic approach and miRNA microarray analysis, we identified 33 potential miRNAs belonging to 27 families in cotton. miRNA microarray and quantitative real-time PCR assays showed that miRNAs expressed differently at the sporogenous cell, pollen mother cell and pollen grain stages between the cotton GMS and its maintainer line. These cotton miRNAs may regulate 35 potential target genes involved in cotton growth and development, signal transduction and metabolism pathways. The expressions of four targets were contrary to the expression of their corresponding miRNAs. These findings enhance our understanding of the roles of miRNAs during fertile and sterile anther development.
\end{abstract}

Keywords: cotton, genetic male sterility, microRNA, differential expression

\section{Introduction}

Male sterility is not only a ubiquitous biological phenomenon, but also an important way to utilize heterosis in plants. The process of pollen development mainly includes the formation of the microspore and male gametogenesis, and is similar throughout higher plant species (Wilson \& Zhang, 2009). Through meiosis, each pollen mother cell can form four microspores in a tetrad. After the microspores lose the thick wall of the tetrad, the free uninucleate microspore undergoes two mitotic divisions to form the male gametophyte also known as a three-celled organism (McCormick, 1993, 2004). Anther development involves a series of complex physiological and biochemical process and, therefore, abnormality at any point in this process can cause male sterility (McCormick, 2004).

MicroRNAs (miRNAs) are a newly discovered class of 19- to 25-nucleotides (nt) small non-coding RNAs that regulate gene expression by mRNA cleavage or translational repression in eukaryotes (Carrington \& Ambros, 2003; Schwab et al., 2005; Axtell et al., 2007). They are processed from their double-stranded precursors by RNase III enzyme Dicer (Cobb et al., 2005). Approaches including cloning, bioinformatics and deep sequencing have been used in various organisms to identify miRNAs. Currently, there are 21,264 miRNA entries in the miRBase (Release 19, August 2012; http://microrna.sanger.ac.uk/), which includes 39 miRNAs identified and confirmed in cotton (Gossypium hirsutum L.). In plants, miRNAs have been shown to play important roles in organismal development, hormonal response, signal transduction and stress response (Pasquinelli et al., 2005; Liu \& Chen 2009; Sunkar, 2010). Many miRNAs have been identified and analyzed in the pollen development of Arabidopsis, rice, maize and hickory in recent years. Chambers and Shuai (2009) identified 37 known miRNAs in Arabidopsis pollen by using miRCURY LNA array technology. Similarly, Grant-Downton et al., (2009) not only detected 33 different miRNA families in mature pollen independently by using quantitative RT-PCR (qRT-PCR) but also identified seven putative novel miRNAs. Peng et al. (2012) identified 47 known and 57 novel miRNAs during male gametophyte development in rice from sRNA-Seq datasets, and also found 
that most of the new miRNAs were pollen-specific and not conserved among species. Seven novel miRNA families and one known miRNA were cloned by constructing a small RNA library from a mixture of anthers from a cytoplasmic male sterile line and its maintainer in maize, all of which showed different expression patterns according to qRT-PCR analysis between the two lines (Shen et al., 2011). In hickory, 39 conserved, two novel and ten potential novel miRNAs were identified by using Solexa technology to sequence two small RNA libraries from two floral differentiation stages (Wang et al., 2012). In addition, there is increasing evidence showing that the function of miRNAs such as miR156, miR159, miR164, miR167, miR172 and miR319 is significant during flower development, from the earliest to very late stages (Wu et al., 2006; Palatnik et al., 2007; Glazinska et al., 2009; Nag et al., 2009). Through researching the mechanism of miRNA action, we can better understand the relationship between miRNA and plant male sterility.

Genetic male sterility (GMS), controlled by nuclear genes, is useful for hybrid seed production in crops. In the past few years, the mechanism of GMS has been intensively studied by using male sterility mutants in cotton. Most research has involved the differences in carbohydrate, free amino acids, enzymes, endogenous hormones and gene expression between the process of fertile and sterile anther development and molecular mapping of genic male-sterile genes (Song et al., 2004; Ma et al., 2007; Chen et al., 2009). With the development of bioinformatics and experimental methods, miRNAs were increasingly identified in cotton (Qiu et al., 2007; Zhang et al., 2007; Li et al., 2012; Yin et al., 2012; Wei et al., 2013). However, to our knowledge, the potential miRNAs involved in cotton anther development remain few.

A cotton 21A male sterility line was found to be under the control of a recessive gene. There was no pollen or a little in the mature anthers of male sterile plants, and no seed set by self-crossing. The microsporocyte development of 21A plant was abnormal at meiosis, and anther abortion occurred mainly at the pollen grain stage. Allelic tests indicated that the male sterility observed in $21 \mathrm{~A}$ was non-allelic to all male sterile genes identified previously in upland cotton (Feng et al., 2010). In this study, 21 A and its maintainer line were used to detect miRNAs and their target genes from cotton anthers using computational approaches. Expression patterns of potential miRNAs and their targets were further analyzed by miRNA microarray assay and qRT-PCR techniques.

\section{Materials and Methods}

\subsection{Plant Materials}

The new upland cotton (G. hirsutum L.) 21A GMS line (SL) and its maintainer line (ML) were grown respectively at different locations of Shandong Agricultural University, Taian, Shandong Province, China. At full bloom stage, developing anthers at the sporogenous cell, pollen mother cell and pollen grain stages (bud lengths $<3.5,3.5-5.0$ and $>5.0 \mathrm{~mm}$, respectively) were separately harvested. The anther samples were quickly frozen in liquid nitrogen and stored at $-80^{\circ} \mathrm{C}$.

\subsection{RNA preparation}

Total RNA was isolated from each sample using the TRIzol ${ }^{\circledR}$ reagent (Invitrogen). The quality of the RNA was assayed using the 2100 Expert_Eukaryotic Total RNA Pico assay.

\section{3 miRNA and Target Gene Prediction}

Computational approaches were used to predict the potential miRNAs and their target genes, using methods described by Yin et al. (2008). The major steps for searching potential miRNAs and target genes in cotton are shown in Supplementary Figure 1. The cotton expressed sequenced tag (EST), genomic survey sequences (GSS) and mRNA databases were obtained from the NCBI databases. To identify new potential miRNAs, all previously known plant miRNA sequences obtained from the miRNA Registry Database (Release 18, http:/www.mirbase.org) were used in BLAST searches against the cotton EST and GSS databases. All BLAST results were saved. The sequences with $\leq 3$ mismatches compared with the query miRNA sequences were chosen manually for further options. The hairpin structures of the selected sequences were predicted using Mfold 3.2. Redundant nucleotides were deleted in order to obtain the mature sequences of predicted miRNAs. To search potential target genes in cotton, the mature miRNA sequences identified were used for a BLAST search in the EST database and genome sequence database of G. Raimondii (Wang et al., 2012). Sequences with $\leq 4$ mismatches, compared with the query miRNA sequences, were chosen. Because the Unigene database contains many transcript sequences that appear to come from the same transcription locus (Yin et al., 2012), we removed redundant sequences.

\section{4 miRNA Microarray Assay}

Microarray assay was performed using a service provider (LC Sciences). To determine differential expression of 
miRNAs between 21A GMS line and its maintainer line samples, 1014 previously known and 33 predicted miRNA probes in triplicate were contained in each miRNA microarray. Three chips were separately used for groups of the sporogenous cell, pollen mother cell and pollen grain stages. The assay started from 2-5 $\mu \mathrm{g}$ of total RNA, which was size fractionated using a YM-100 Microcon centrifugal filter (Millipore) and the small RNAs $(<300 \mathrm{nt})$ isolated were 3'-extended with a poly(A) tail using poly(A) polymerase. An oligonucleotide tag was then ligated to the poly(A) tail for later fluorescent dye staining. Hybridization was performed overnight on a $\mu$ Paraflo $^{\mathrm{TM}}$ microfluidic chip using a micro-circulation pump (Atactic Technologies). The hybridization melting temperatures were balanced by chemical modifications of the detection probes. Hybridization used $100 \mu \mathrm{L}$ of $6 \times$ SSPE buffer $\left(0.90 \mathrm{M} \mathrm{NaCl}, 60 \mathrm{mM} \mathrm{Na}_{2} \mathrm{HPO}_{4}\right.$ and $6 \mathrm{mM}$ EDTA at $\left.\mathrm{pH} 6.8\right)$ containing $25 \%$ formamide at $34{ }^{\circ} \mathrm{C}$. After hybridization, detection was by fluorescence labeling using tag-specific Cy 3 and Cy5 dyes. Hybridization images were collected using a laser scanner (GenePix 4000B, Molecular Device) and digitized using Array-Pro image analysis software (Media Cybernetics). Data were analyzed by first subtracting the background and then normalizing the signals using a LOWESS filter. For two color experiments, the ratio of the two sets of detected signals ( $\log _{2}$ transformed, balanced) and p-values of the $t$-test were calculated; differentially detected signals were those with $\mathrm{p}<0.01$.

\section{5 qRT-PCR Analysis}

qRT-PCR was used to analyze the relative expression of miRNAs and target genes. The specific miRNAs and target genes primers used are given in Supplementary Table 1. For qRT-PCR of miRNAs, however, Uni-miR qPCR Primer (TAKARA, Dalian, China) was used instead of the PCR forward primer. U6 small nuclear RNA served as an internal standard in miRNA amplification experiments, and Ubiquitin gene (UBI) in experiments of gene qRT-PCR. For the first-strand cDNA synthesis experiment, One Step PrimeScript ${ }^{\circledR}$ miRNA cDNA Synthesis Kit (TAKARA) was used following the manufacturer's instruction. From each sample, $4 \mu \mathrm{g}$ of total RNA was converted to cDNA in a $20-\mu \mathrm{L}$ reaction system which contained $10 \mu \mathrm{L}$ of $2 \times$ miRNA reaction buffer mix, $2 \mu \mathrm{L}$ of $0.1 \%$ BSA and $2 \mu \mathrm{L}$ of miRNA PrimeScript ${ }^{\circledR}$ RT Enzyme Mix. qRT-PCR for miRNAs and target genes was performed using SYBR ${ }^{\circledR}$ Premix Ex Taq ${ }^{\mathrm{TM}}$ II (TAKARA) and carried out in Bio-RAD iCycler iQ5 Machine with following cycling profile: $95^{\circ} \mathrm{C}$ at $30 \mathrm{~s}$, followed by 40 cycles of $95{ }^{\circ} \mathrm{C}$ at $15 \mathrm{~s}$ and $62{ }^{\circ} \mathrm{C}$ at $30 \mathrm{~s}$. The reaction mixture was prepared in triplicate. Each $25-\mu \mathrm{L}$ reaction mixture contained $12.5 \mu \mathrm{L}$ of SYBR ${ }^{\circledR P r e m i x}$ Ex TaqTM II $(2 \times), 1 \mu \mathrm{L}$ of PCR forward primer $(10 \mu \mathrm{M}), 1 \mu \mathrm{L}$ of PCR reverse primer $(10 \mu \mathrm{M})$ and $2 \mu \mathrm{L}$ of fivefold diluted cDNA template. After reactions were completed, the $2^{-\Delta \Delta \mathrm{Ct}}$ method $[\Delta \mathrm{Ct}=\mathrm{Ct}$ (miRNA or target gene) $-\mathrm{Ct}$ (U6 or UBI), $\Delta \Delta \mathrm{Ct}=\Delta \mathrm{Ct}(\mathrm{SL})-\Delta \mathrm{Ct}(\mathrm{ML}), 2^{-\Delta \Delta \mathrm{Ct}}=$ Relative Expression] was used to calculate relative expression of miRNAs or target genes (Livak et al., 2001).

\section{Results}

\subsection{Identification of potential cotton miRNAs}

In our study, a homology search was used to identify conserved miRNAs in cotton. Following a set of strict filtering criteria, a total of 33 cotton miRNAs belonging to 27 miRNA families were identified in cotton EST and GSS databases (Table 1). The sequences of these newly identified miRNAs were 20-22 nt in length. A total of 24 miRNA mature sequences began with 5'-uridine a characteristic of plant miRNAs. In addition, the length of miRNA precursors was $48-513 \mathrm{nt}$, but a majority of them (72.7\%) required $65-151 \mathrm{nt}$, which is similar to that observed in many other species (Nasaruddin et al., 2007; Yin et al., 2008). Furthermore, A + U contents of those pre-miRNAs sequences were calculated in the range of 36.76-74.77\%. All of those miRNA precursors can be folded to the typical hairpin structure of the miRNA family (Supplementary Figure 2). The minimal folding free energy index (MFEI) is an available standard for distinguishing miRNAs from other types of coding or non-coding RNAs. In our results, the average MFEIs of the 33 pre-miRNAs was 0.81; a majority of the identified cotton pre-miRNAs had MFEI values in the range 0.71-1.29, which is remarkably higher than that for tRNAs (0.64), rRNAs (0.59) and mRNAs (0.62-0.66) (Zhang et al., 2006). For the 33 identified potential miRNAs, miR156, miR395, miR414 and miR426 had 2-3 family members each, while the remainder had only one member. The miR426 family and the other miRNAs, including miR403, miR418, miR477, miR529, miR834, miR846, miR857, miR1044 and miR1063 were identified in cotton for the first time. 
Table 1. Newly identified miRNAs from cotton

\begin{tabular}{|c|c|c|c|c|c|c|}
\hline miRNAs & Gene ID & Sequence $\left(5^{\prime}\right.$ to $\left.3^{\prime}\right)$ & $\begin{array}{l}\text { Gene } \\
\text { source }\end{array}$ & $\begin{array}{l}\mathrm{A}+\mathrm{U} \\
(\%)\end{array}$ & MFEIs & $\begin{array}{l}\mathrm{LP} \\
\text { (nt) }\end{array}$ \\
\hline ghr-miR156 & DX535560 & UAGCAGAAGAAAGAGAGCAU & $5^{\prime} / \mathrm{GSS}$ & 69.81 & 0.65 & 53 \\
\hline ghr-miR156a & DW508467 & UGACAGAAGAGAGUGAGCAC & $5^{\prime} / \mathrm{EST}$ & 48.78 & 1.29 & 83 \\
\hline ghr-miR156b & DT558905 & UGACAGAAGAGAGAGAGCACG & $5^{\prime} / \mathrm{EST}$ & 56.00 & 0.63 & 151 \\
\hline ghr-miR162 & DW493971 & UCGAUAAACCUCUGCAUCCAG & $5^{\prime} / \mathrm{EST}$ & 57.14 & 0.91 & 91 \\
\hline ghr-miR164 & DR461140 & UGGAGAAGCAGGGCACGUGCA & $5^{\prime} / \mathrm{EST}$ & 48.48 & 1.13 & 65 \\
\hline ghr-miR166 & DW502146 & UCGGACCAGGCUUCAUUCCCC & $5^{\prime} / \mathrm{EST}$ & 56.46 & 0.95 & 147 \\
\hline ghr-miR167 & DW507007 & ACAGCUGCCAUCAUGAUCUUG & $5^{\prime} / \mathrm{EST}$ & 58.33 & 0.55 & 48 \\
\hline ghr-miR171 & DW507416 & AGAUUGAGCCGCGCCAAUAUC & $5^{\prime} / \mathrm{EST}$ & 56.47 & 1.02 & 85 \\
\hline ghr-miR390 & DW518164 & AAGCUCAGGAGGGAUAGCGCC & $5^{\prime} / \mathrm{EST}$ & 57.29 & 0.98 & 96 \\
\hline ghr-miR393 & DR461688 & UCCAAAGGGAUCGCAUUGAUCU & $5^{\prime} / \mathrm{EST}$ & 61.34 & 0.98 & 119 \\
\hline ghr-miR394 & DW517361 & UUGGCAUUCUGUCCACCUCC & $5^{\prime} / \mathrm{EST}$ & 60.20 & 0.73 & 98 \\
\hline ghr-miR395a & DT567568 & UUGAAGUGUUUGGGGGAACUU & $5^{\prime} / \mathrm{EST}$ & 59.14 & 0.75 & 186 \\
\hline ghr-miR395c & DX556947 & CUAAAGUGUUUAGGGGAACUC & $5^{\prime} / \mathrm{GSS}$ & 0.619 & 1.04 & 84 \\
\hline ghr-miR395d & DX543072 & CUGAAGUGUUUGGGGGAACUC & $5^{\prime} / \mathrm{GSS}$ & 52.33 & 1.06 & 118 \\
\hline ghr-miR396 & DW501664 & UUCCACAGCUUUCUUGAACUG & $5^{\prime} / \mathrm{EST}$ & 60.00 & 0.91 & 115 \\
\hline ghr-miR398 & DW498056 & UGUGUUCUCAGGUCACCCCUU & $5^{\prime} / \mathrm{EST}$ & 49.25 & 0.96 & 67 \\
\hline ghr-miR399 & DW509341 & UGCCAAAGGAGAUUUGCCCU & $5^{\prime} / \mathrm{EST}$ & 60.00 & 1.23 & 77 \\
\hline ghr-miR403 & DW507201 & UUAGAUUCACGCACAAAGCCC & $5^{\prime} / \mathrm{EST}$ & 54.43 & 0.60 & 79 \\
\hline ghr-miR408 & DW518984 & GGCACUGCGUCUUCCCUGGAU & $5^{\prime} / \mathrm{EST}$ & 36.76 & 0.81 & 69 \\
\hline ghr-miR414 & DW242385 & UCAUCCUCAUAAUCCUCGUGC & $5^{\prime} / \mathrm{EST}$ & 49.32 & 0.57 & 513 \\
\hline ghr-miR414b & DT564396 & UUUUCUUCAUCAUCAUCGUCA & $5^{\prime} / \mathrm{EST}$ & 53.52 & 0.62 & 142 \\
\hline ghr-miR418 & DX527779 & UAAUGUGAUGAUUAAAUGGGU & $5^{\prime} / \mathrm{GSS}$ & 68.91 & 0.55 & 431 \\
\hline ghr-miR426a & DX548009 & UUUUGGAAAUUAGUCCUUGGG & $5^{\prime} / \mathrm{GSS}$ & 70.00 & 0.57 & 150 \\
\hline ghr-miR426b & DX551631 & UUUUGCAAAUUAGUCCUUAGG & $5^{\prime} / \mathrm{GSS}$ & 70.00 & 0.53 & 150 \\
\hline ghr-miR477 & DX525152 & CUCUCCCUCAAGGGCUUCCC & $5^{\prime} / \mathrm{GSS}$ & 49.43 & 0.73 & 87 \\
\hline ghr-miR482 & DR457519 & UCUUUCCUACUCCUCCCAUACC & $5^{\prime} / \mathrm{EST}$ & 60.00 & 0.96 & 85 \\
\hline ghr-miR529 & DW517772 & GGAAAGAGAGAGAGAACAGC & $5^{\prime} / \mathrm{EST}$ & 60.00 & 0.67 & 272 \\
\hline ghr-miR827 & DW520471 & UUAGAUGACCAUCAACAAACA & $5^{\prime} / \mathrm{EST}$ & 63.41 & 0.86 & 123 \\
\hline ghr-miR834 & DX522699 & UGGTAGCAGCAGUGGUGGUGC & $5^{\prime} / \mathrm{GSS}$ & 53.95 & 0.71 & 304 \\
\hline ghr-miR846 & DW243653 & UUGAAUGGAAGUGCUUGACAU & $5^{\prime} / \mathrm{EST}$ & 54.55 & 0.73 & 65 \\
\hline ghr-miR857 & DX531959 & CUUUGUAUGGUGAAGGUGUAA & $5^{\prime} / \mathrm{GSS}$ & 64.65 & 0.61 & 199 \\
\hline ghr-miR1044 & DR462558 & UUGUAGUUCAUAUUUGUAUG & $5^{\prime} / \mathrm{EST}$ & 74.77 & 0.90 & 111 \\
\hline ghr-miR1063 & DW511180 & UCUCUUGGAGCACUGCAUCUG & $5^{\prime} / \mathrm{EST}$ & 63.95 & 0.60 & 61 \\
\hline
\end{tabular}

MFEIs, minimal folding free energy indexes; LP: length of miRNA precursor.

\subsection{Microarray Analysis}

Microarray technique was used to profile global miRNA expression between the 21A GMS line and its maintainer line during anther development. To confirm the predicted cotton miRNAs above, 33 probes complementary to the potential miRNAs were designed and assigned to each chip. Microarray data indicated that 504, 432 and 439 transcripts were detected perfectly at the sporogenous cell, pollen mother cell and pollen grain 
stages of anther development, respectively. Among them, all 33 potential cotton miRNAs were detected in both groups; 10 were differentially expressed at the three stages of anther development using miRNA microarray (Figure 1).

Analysis of microarray data showed that most miRNAs of miR156, miR164 and miR166 families were up-regulated in the SL compared with the ML group at the three stages of anther development. Compared with the ML group, miR159, miR172 and miR319 families were up-regulated at the sporogenous cell stage, but down-regulated at the pollen mother cell and pollen grain stages in the SL group (Figure 1). These data suggested that anther development involved a series of expressions of conserved miRNAs. The regulation of these miRNAs from other species implied that they might play significant roles during anther development in cotton.

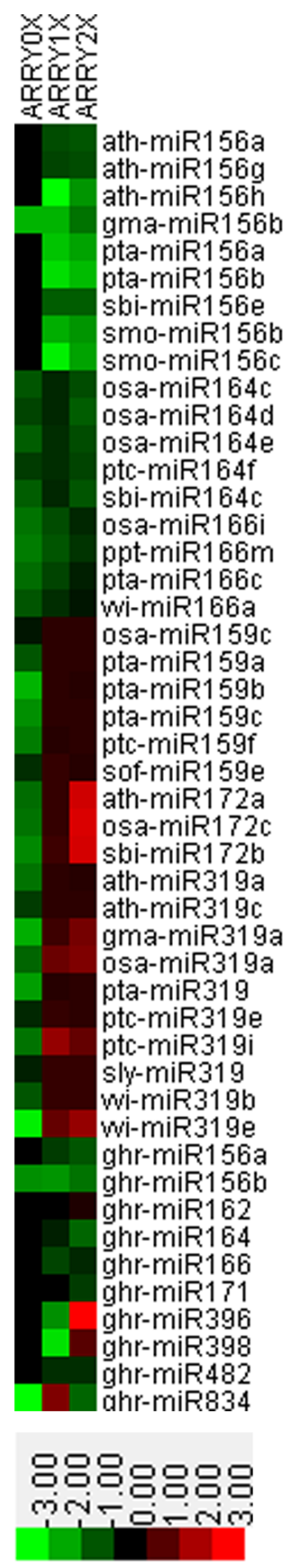

Figure 1. Expression profiles of miRNAs in different anther developmental stages between cotton 21A genetic male sterile line (SL) and its maintainer line (ML) using microarrays 
Each row represents a miRNA and each column represents a stage of anther development. ARRYOX sporogenous cell, $A R R Y 1 X$ pollen mother cell and $A R R Y 2 X$ pollen grain stages. The clustering is performed on $\log _{2}$ (ML/SL) ratio on variation across samples. The intensities of the color represent the relative magnitude of fold changes in log values: -3.0 indicates that the miRNA is highly suppressed; while +3.0 indicates that it is highly induced.

\section{3 miRNA Expression Profiling With qRT-PCR}

To validate the microarray data, 12 miRNAs were selected at random for qRT-PCR analysis for the relative expression of miRNAs at the three stages of the cotton anther development respectively. qRT-PCR analysis showed that the expression of 10 miRNAs fitted well with the pattern of microarray analysis (Figure 2). However, there was no obvious differential expression between the SL and ML samples for ghr-miR156b at the sporogenous cell stage.

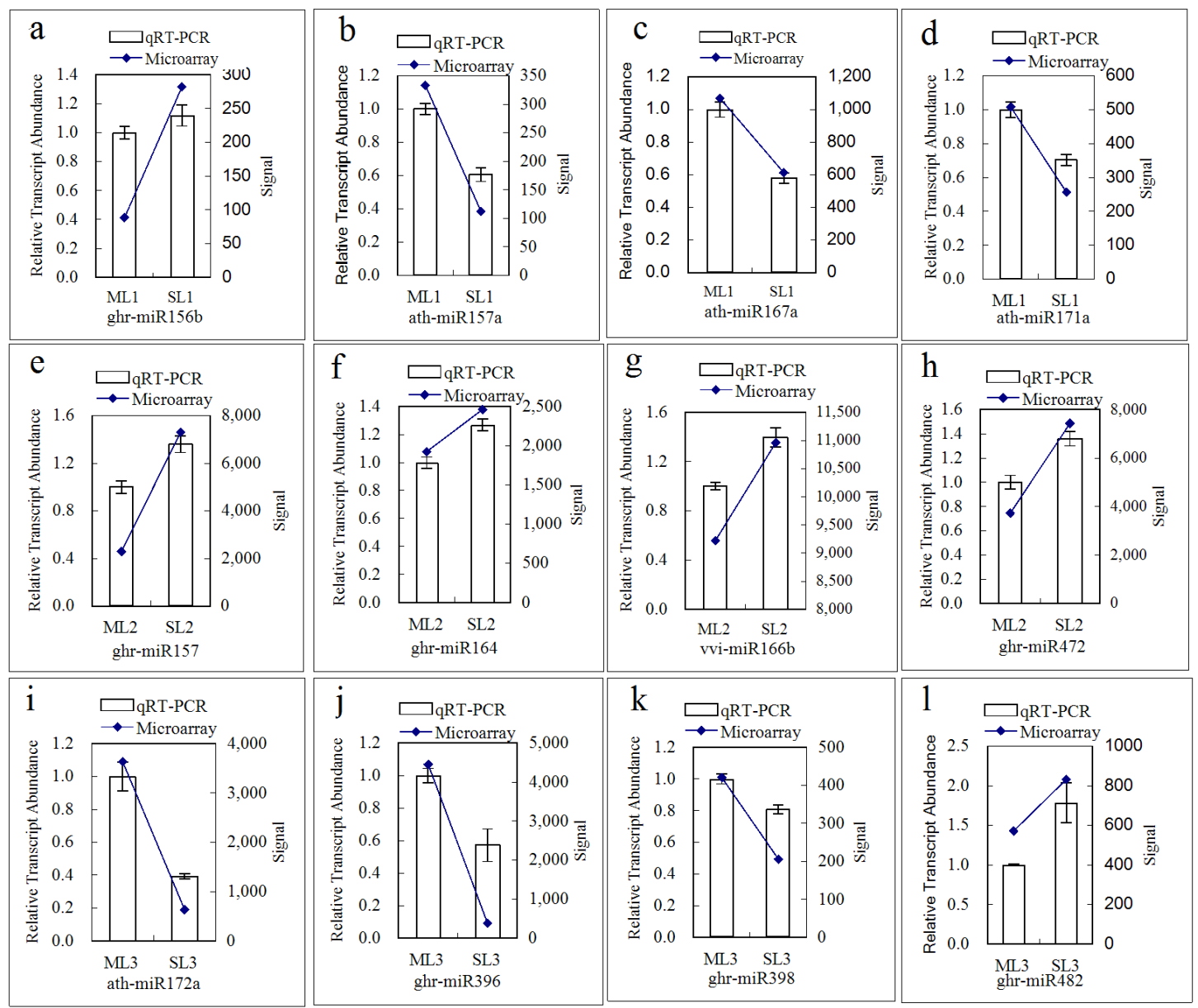

Figure 2. qRT-PCR validation of conserved miRNAs from cotton anthers

SL, 21A genetic male sterile line; ML, maintainer line; 1: sporogenous cell stage; 2: pollen mother cell stage; 3: pollen grain stage (a-d, Expression of miRNAs at the sporogenous cell stage; e-h, expression of miRNAs at the pollen mother cell stage; i-1, expression of miRNAs at the pollen grain stage).

To further determine the relative expression of identified new cotton miRNAs at the different stages of anther development, qRT-PCR was also used for analysis - the expression patterns of these miRNAs were intriguing. Both in the SL and ML samples: (1) the expression levels of ghr-miR156a, ghr-miR156b, ghr-miR171, ghr-miR396 and ghr-miR398 had no significant differences between the sporogenous cell and pollen mother cell stages, but were up-regulated remarkably at the pollen grain stage; (2) the ghr-miR164, ghr-miR166 and ghr-miR482 showed decreased expression at the pollen mother cell compared to the other two stages; and (3) there was no dramatic differential expression for both ghr-miR162 and ghr-miR834 in these three stages. In 
addition, qRT-PCR analysis also showed no obvious differential expression for some miRNAs between the SL and ML samples, but a high expression for ghr-miR156a, ghr-miR156b, ghr-miR164, ghr-miR166, ghr-miR171 and ghr-miR482 at the pollen grain stage, and a low expression for ghr-miR398 at these three stages in the SL sample. The experimental data show that these miRNAs were differentially regulated at different stages of the cotton anthers between the GMS and the maintainer lines (Figure 3). Therefore, these differentially expressed miRNAs might play important roles in the process of anther development.

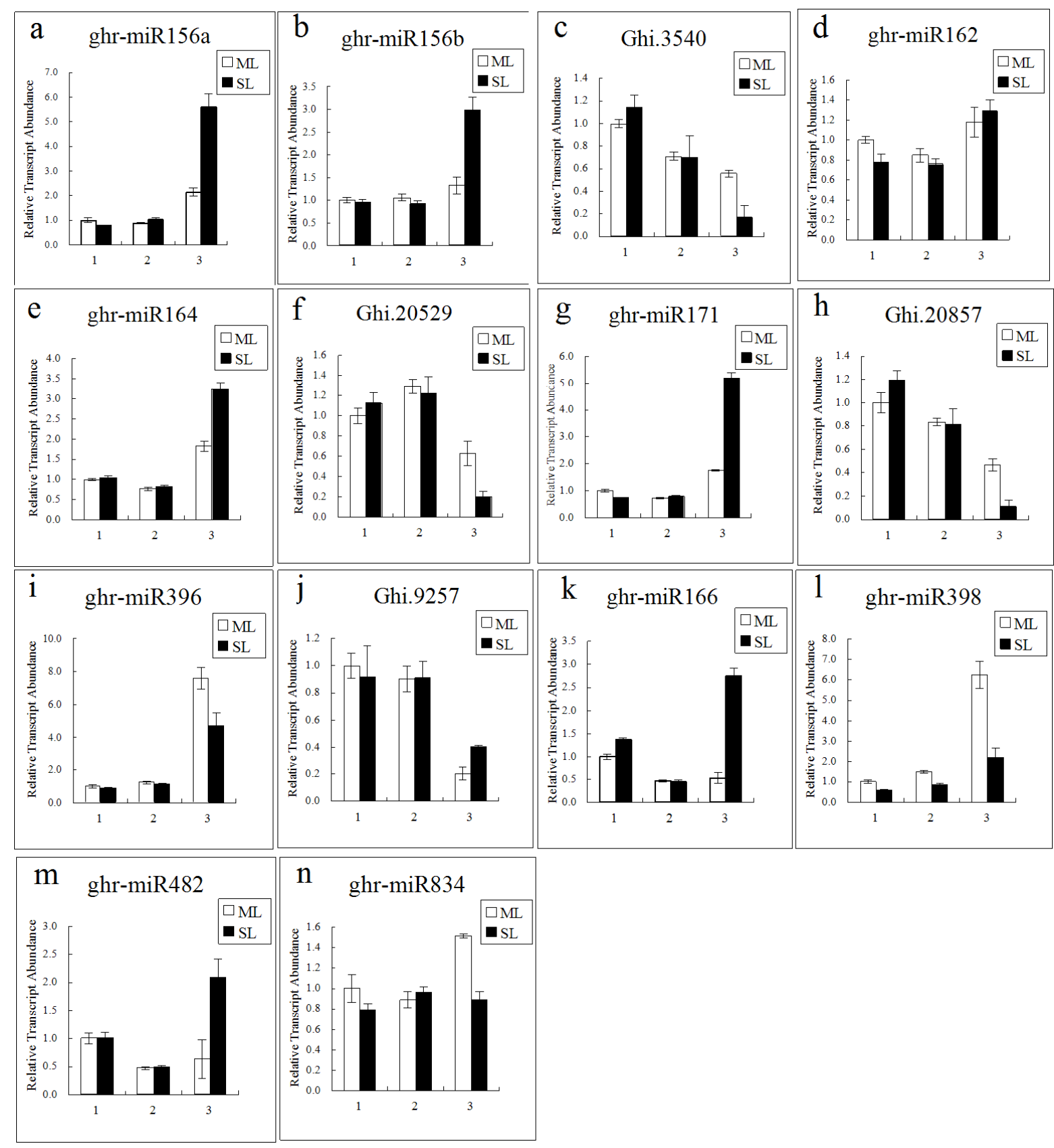

Figure 3. Differential expression of miRNAs and target genes between cotton 21A genetic male sterile line (SL) and its maintainer line (ML) with the development of anther

1: sporogenous cell stage; 2: pollen mother cell stage; 3: pollen grain stage (a b d e g i k-n, expression patterns of 10 miRNAs during the cotton anther development between the GMS line and its maintainer; $\mathrm{c} \mathrm{f} \mathrm{h} \mathrm{j}$, expression patterns of four target genes during the cotton anther development between the GMS and its maintainer line). Ghi.3540, squamosa-promoter-binding-like protein 10 targeted by ghr-miR156b; Ghi.20529, NAC domain protein targeted by ghr-miR164; Ghi.20857, scarecrow-like (SCL) protein 22 targeted by 
ghr-miR171; Ghi.9257, growth-regulating factor targeted by ghr-miR396.

\subsection{Target Genes of miRNAs and Their Expression Analysis of qRT-PCR Assay}

The newly identified miRNAs were used as query sequences for BLASTn searches to identify miRNA targets in the cotton mRNA database. In this study, we identified a total of 35 potential target genes for 20 of the new miRNAs from the cotton mRNA database (Supplementary Table 2). To examine the functional similarity of predicted targets, the cotton entries were selected from the Unigene database. Annotated mRNA targets had various biological functions in plant anther development, with the majority related to growth and development, transcriptional factors and metabolic pathways. Up to now, there have also been several predicted targets encoding unknown proteins. In this study, eight identified miRNA families in cotton targeted a variety of transcription factors, which play important roles in cotton anther development. miR156 targets the squamosa-promoter binding protein transcription factors and squamosa-promoter binding protein-like (SPL) genes. miR164 targets NAC transcription factor, and miR167 targets auxin response factors that play roles in plant signaling transduction and root development. It is interesting to note that those transcription factors are similar to those reported previously as miRNA targets for other plant species (Mallory et al., 2004; Arazi et al., 2005; Wu et al., 2006; Yang et al., 2006; Fu et al., 2012). In this study, we also found that scarecrow-like (SCL) protein 22 and mitochondrial transcription termination factor have near-perfect complementary sites with miR171 and miR426, respectively.

To confirm the prediction of the target genes, we performed qRT-PCR analysis of several target genes between the fertile and sterile lines. The mRNAs of four targets tested showed expression patterns of the potential target genes contrary to the expression of their corresponding miRNAs (Figure 3). Therefore, our predictions of the partial target genes were preliminarily verified by qRT-PCR. Further research is needed to verify these target genes using 5 ' rapid amplification of cDNA ends.

\section{Discussion}

miRNAs, such as miR156, miR159, miR164, miR166, miR172 and miR319 play important roles during the development of flower organs and some participate in the process of male sterility. In this study, using bioinformatic and miRNA microarray approaches, 33 miRNAs were identified in cotton anther tissue. The miR426 family and the other miRNAs, including miR403, miR418, miR477, miR529, miR834, miR846, miR857, miR1044 and miR1063 were firstly identified in cotton. qRT-PCR showed that 10 miRNAs and their targets had a specific expression pattern differences between the GMS and maintainer lines during the three stages of cotton anther development.

During the process of fertile and sterile anther development, two miR156s, miR171, miR396 and miR398 were up-regulated remarkably at the pollen grain stage, but down-regulated at the sporogenous cell and pollen mother cell stages. Moreover, miR156s and miR171 were up-regulated at the pollen grain stage of sterile anther development, but miR396 down-regulated. Specifically for miR398, expression decreased at the three stages of sterile anther development. In some plant species, miR156s, miR171 and miR396 have been shown to target SPL transcription factors, SCL transcription factors and growth-regulating factors (GRF), respectively (Llave et al., 2002; Liu et al., 2009; Wang et al., 2009; Wang et al., 2011). The functions of these targets were found to be related to flower development, e.g. controlling flowering time (Llave et al., 2002; Xie et al., 2006). GRFs, a plant specific family of transcription factors known to control cell proliferation in leaves (Rodriguez et al., 2010), especially also play important regulatory roles in floral organ development. Reduced expression of NtGRF-like genes was reported to result in abnormalities such as many more petals, stamens and carpels, but lower fertility than in wild-type tobacco flowers (Yang et al., 2009). miR398 has been shown to target COX5b.1, two copper superoxide dismutase (CSD) enzymes and copper chaperone for superoxide dismutase (CCS1) in Arabidopsis (Jones-Rhoades and Bartel, 2004; Sunkar and Zhu, 2004; Bouché, 2010). Those targets were involved in different abiotic stresses. However, miR398 has not been previously reported to be related to male sterility. Significantly, we observed that expression of miR398 was down-regulated during the process of sterile anther development, suggesting that miR398 may be strongly related to male sterility.

miR164, miR166 and miR482 showed decreased expression from the pollen mother cell to the sporogenous cell stage, but increased from the pollen grain to the pollen mother cell stage in the GMS and maintainer lines; significantly, there was high expression for these miRNAs in sterile tissue at the pollen grain stage. Recent studies demonstrated that miR164, miR166 and miR482 target NAC transcription factor family genes, Class III HD-ZIP transcription factor family genes and nucleotide-binding site leucine-rich repeat (NB-LRR) genes, respectively (Emery et al., 2003; Mallory et al., 2004; Ko et al., 2006). Analysis of early extra petals1 
Arabidopsis mutants confirmed that miR164c prevents extra petals in early-arising flowers by repressing CUC1 and CUC2, which were members of NAC transcription factor family (Baker et al., 2005). In addition, it was shown that miR166 regulated vascular development in Arabidopsis inflorescence stems through directed cleavage of ATHB15 mRNA (Kim et al., 2005), one of the predicted targets of miR166 in the present study. Through the phenotypes of a series of double mutants, the miR166/165 signaling pathways function in parallel to the WUS-CLV pathway in regulating the shoot apical meristem and floral development (Jung \& Park, 2007). Eitas and Dangl (2010) found that NB-LRR proteins functioned in microbial recognition as activators of defense responses in plants. However, the function of protein encoded by targets of miR482 remained unknown in our prediction results. Both in the GMS and its maintainer line, there was no dramatic differential expression for miR162 and miR834 during anther development. These data suggest that miR162 and miR834 were not related to fertile and sterile anther development.

In conclusion, we identified 33 miRNAs belonging to 27 families from cotton EST and GSS databases based on bioinformatics and microarray analysis. Ten miRNA families were observed for the first time in cotton. These new miRNAs are highly conserved among plant species and some showed diverse expression patterns during the cotton anther development between the GMS and its maintainer line. We also obtained 35 potential miRNA targets which included transcription factors regulating plant growth and development, enzymes and proteins involved in cotton metabolism and signal transduction, implying that miRNA may play important roles in cotton anther development. Further research on function analysis of the target genes would give a better understanding of the anther abortion mechanisms of the GMS line in cotton.

\section{References}

Arazi T., Talmor-Neiman M., Stav R., Riese M., Huijser P., \& Baulcombe, D. C. (2005). Cloning and characterization of micro-RNAs from moss. The Plant journal : for cell and molecular biology, 43(6), 837-848. http://dx.doi.org/10.1111/j.1365-313X.2005.02499.x

Axtell, M. J., Snyder, J. A., \& Bartel, D. P. (2007). Common functions for diverse small RNAs of land plants. The Plant cell, 19(6), 1750-1769. http://dx.doi.org/10.1105/tpc.107.051706

Baker, C. C., Sieber, P., Wellmer F., \& Meyerowitz, E. M. (2005). The early extra petals1 mutant uncovers a role for microRNA miR164c in regulating petal number in Arabidopsis. Curr Biol, 15(4), 303-315. http://dx.doi.org/10.1016/j.cub.2005.02.017

Bouché, N. (2010). New insights into miR398 functions in Arabidopsis. Plant Signaling \& Behavior, 5(6), 684-686. http://dx.doi.org/10.1111/j.1365313X.2010.04162.x

Carrington, J. C., \& Ambros, V. (2003). Role of microRNAs in plant and animal development. Science, 301(5631), 336-338. http://dx.doi.org/10.1126/science.1085242

Chambers, C., \& Shuai, B. (2009). Profiling microRNA expression in Arabidopsis pollen using microRNA array and real-time PCR. BMC plant biology, 9, 87. http://dx.doi.org/10.1186/1471-2229-9-87

Chen, D., Ding, Y., Guo, W., \& Zhang, T. (2009). Molecular mapping of genic male-sterile genes $m s_{15}, m s_{5}$ and $m_{6}$ in tetraploid cotton. Plant Breeding, 128(2), http://dx.doi.org/10.1111/j.1439-0523.2008.01562.x

Cobb, B. S., Nesterova, T. B., Thompson, E., Hertweck, A., O'Connor, E., Godwin, J., ... Merkenschlager, M. (2005). T cell lineage choice and differentiation in the absence of the RNase III enzyme Dicer. $J$ Exp Med, 201(9), 1367-1373. http://dx.doi.org/10.1084/jem.20050572

Eitas, T. K., \& Dangl, J. L. (2010). NB-LRR proteins: pairs, pieces, perception, partners, and pathways. Current opinion in plant biology, 13(1), 472-477. http://dx.doi.org/10.1016/j.pbi.2010.04.007

Emery, J. F., Floyd, S. K., Alvarez, J., Eshed, Y., Hawker, N. P., Izhaki, A., ... Bowman, J. L. (2003). Radial patterning of Arabidopsis shoots by class III HD-ZIP and KANADI genes. Curr Biol, 13(20), 1768-1774. http://dx.doi.org/10.1016/j.cub.2003.09.035

Feng, X., Liu, F., Liu, Y., Yin, Z., Han, X., \& Shen, F. (2010). Studies of New Genic Male Sterility Cotton Named 21A in Upland Cotton (Gossypium hirsutum L.). Journal of Plant Genetic Resources, 11(4), 433-438.

Fu, C., Sunkar, R., Zhou, C., Shen, H., Zhang, J. Y., Matts, J., ... Wang, Z. Y. (2012). Overexpression of miR156 in switchgrass (Panicum virgatum L.) results in various morphological alterations and leads to improved $\begin{array}{llllll}\text { biomass } & \text { production. } & \text { Plant } & \text { Biotechnol } & \text { 40(4), } & \text { 443-452. }\end{array}$ http://dx.doi.org/10.1111/j.1467-7652.2011.00677.x 
Glazinska, P., Zienkiewicz, A., Wojciechowski, W., \& Kopcewicz, J. (2009). The putative miR172 target gene InAPETALA2-like is involved in the photoperiodic flower induction of Ipomoea nil. J Plant Physiol, 166(16), 1801-1813. http://dx.doi.org/10.1016/j.jplph.2009.05.011

Grant-Downton, R., Le Trionnaire, G., Schmid, R., Rodriguez-Enriquez, J., Hafidh, S., Mehdi, S., ... Dickinson, H. (2009). MicroRNA and tasiRNA diversity in mature pollen of Arabidopsis thaliana. BMC genomics, 10, 643. http://dx.doi.org/10.1186/1471-2164-10-643

Jones-Rhoades, M. W., \& Bartel, D. P. (2004). Computational identification of plant microRNAs and their targets, including a stress-induced miRNA. Molecular cell, 14(6), 787-799. http://dx.doi.org/10.1016/j.molcel.2004.05.027

Jung, J. H., \& Park, C. M. (2007). MIR166/165 genes exhibit dynamic expression patterns in regulating shoot apical meristem and floral development in Arabidopsis. Planta, 225(6), 1327-1338. http://dx.doi.org/10.1007/s00425-006-0439-1

Kim, J., Jung, J. H., Reyes, J. L., Kim, Y. S., Chung, K. S., ... Park, C. M. (2005). microRNA-directed cleavage of ATHB15 mRNA regulates vascular development in Arabidopsis inflorescence stems. The Plant journal: for cell and molecular biology, 42(1), 84-94. http://dx.doi.org/10.1111/j.1365-313X.2005.02354.x

Ko, J. H., Prassinos, C., \& Han, K. H. (2006). Developmental and seasonal expression of PtaHB1, a Populus gene encoding a class III HD-Zip protein, is closely associated with secondary growth and inversely correlated with the level of microRNA (miR166). New Phytol, 169(3), 469-478. http://dx.doi.org/10.1111/j.1469-8137.2005.01623.x

Li, Q., Jin, X., \& Zhu, Y. X. (2012). Identification and analyses of miRNA genes in allotetraploid Gossypium hirsutum fiber cells based on the sequenced diploid G. raimondii genome. J Genet Genomics, 39(7), 351-360. http://dx.doi.org/10.1016/j.jgg.2012.04.008

Liu, D., Song, Y., Chen, Z., \& Yu, D. (2009). Ectopic expression of miR396 suppresses GRF target gene expression and alters leaf growth in Arabidopsis. Physiol Plant, 136(2), 223-236. http://dx.doi.org/10.1111/j.1399-3054.2009.01229.x

Liu, Q., \& Chen, Y. Q. (2009). Insights into the mechanism of plant development: interactions of miRNAs pathway with phytohormone response. Biochem Biophys Res Commun, 384(1), 1-5. http://dx.doi.org/10.1016/j.bbrc.2009.04.028

Livak, K. J., \& Schmittgen, T. D. (2001). Analysis of relative gene expression data using real-time quantitative PCR and the $2^{-\Delta \Delta \mathrm{Ct}}$ Method. Methods, 25(4), 402-408. http://dx.doi.org/10.1006/meth.2001.1262

Llave, C., Xie, Z., Kasschau, K. D., \& Carrington, J. C. (2002). Cleavage of Scarecrow-like mRNA targets directed by a class of Arabidopsis miRNA. Science, 297(5589), 2053-2056. http://dx.doi.org/10.1126/science.1076311

Ma, X., Xing, C., Guo, L., Gong, Y., Wang, H., Zhao, Y., \& Wu, J. (2007). Analysis of Differentially Expressed Genes in Genic Male Sterility Cotton (Gossypium hirsutum L.) Using cDNA-AFLP. Journal of Genetics and Genomics, 34(6), 536-543. http://dx.doi.org/10.1016/s1673-8527(07)60059-9

Mallory, A. C., Dugas, D. V., Bartel, D.P ., \& Bartel, B. (2004). MicroRNA regulation of NAC-domain targets is required for proper formation and separation of adjacent embryonic, vegetative, and floral organs. Curr Biol, 14(12), 1035-1046. http://dx.doi.org/10.1016/j.cub.2004.06.022

Mccormick, S. (1993). Male Gametophyte Development. The Plant Cell, 5(10), 1265-1275. http://dx.doi.org/10.1105/tpc.5.10.1265

McCormick, S. (2004). Control of male gametophyte development. The Plant Cell, 16 Suppl, S142-153. $\mathrm{http}: / / \mathrm{dx}$.doi.org/10.1105/tpc.016659

Nag, A., King, S., \& Jack, T. (2009). miR319a targeting of TCP4 is critical for petal growth and development in Arabidopsis. Proc Natl Acad Sci U S A, 106(52), 22534-22539. http://dx.doi.org/10.1073/pnas.0908718106

Nasaruddin, N., Harikrishna, K., Othman, R., Lim, S., \& Hari-Krishna, J. (2007). Computational prediction of microRNAs from oil palm (Elaeis guineensis Jacq.) expressed sequence tags. Asia Pac J Mol Biol Biotechnol, 15(3), 107-113

Palatnik, J. F., Wollmann, H., Schommer, C., Schwab, R., Boisbouvier, J., Rodriguez, R., ... Weigel D. (2007). Sequence and expression differences underlie functional specialization of Arabidopsis microRNAs miR159 and miR319. Dev Cell, 13(1), 115-125. http://dx.doi.org/10.1016/j.devcel.2007.04.012 
Pasquinelli, A. E., Hunter, S., \& Bracht, J. (2005). MicroRNAs: a developing story. Curr Opin Genet Dev, 15(2), 200-205. http://dx.doi.org/10.1016/j.gde.2005.01.002

Peng, H., Chun, J., Ai, T. B., Tong, Y. A., Zhang, R., Zhao, M. M., ... Wang, S. H. (2012). MicroRNA profiles and their control of male gametophyte development in rice. Plant Mol Biol, 80(1), 85-102. http://dx.doi.org/10.1007/s11103-012-9898-x

Qiu, C. X., Xie, F. L., Zhu, Y. Y., Guo, K., Huang, S. Q., Nie, L., \& Yang, Z. M. (2007). Computational identification of microRNAs and their targets in Gossypium hirsutum expressed sequence tags. Gene, 395(1-2), 49-61. http://dx.doi.org/10.1016/j.gene.2007.01.034

Rodriguez, R. E., Mecchia, M. A., Debernardi, J. M., Schommer, C., Weigel, D., \& Palatnik, J. F. (2010). Control of cell proliferation in Arabidopsis thaliana by microRNA miR396. Development, 137, 103-112. http://dx.doi.org/10.1242/dev.043067

Schwab, R., Palatnik, J. F., Riester, M., Schommer, C., Schmid, M., \& Weigel, D. (2005). Specific effects of microRNAs on the plant transcriptome. Dev Cell, 8(4), 517-527. http://dx.doi.org/10.1016/j.devcel.2005.01.018

Shen, Y., Zhang, Z., Lin, H., Liu, H., Chen, J., Peng, H., ... Pan, G. (2011). Cytoplasmic male sterility-regulated novel microRNAs from maize. Funct Integr Genomics, 11(1), 179-191. http://dx.doi.org/10.1007/s10142-010-0202-3

Song, X., Sun, X., Wang, H., Liu, Y., \& Zhang J. (2004). Biochemical changes in anthers of " Dong A" genetic male sterile lines of cotton. Acta Botania Boreali-Occidentalia Sinica, 24(2), 243-247

Sunkar, R. (2010). MicroRNAs with macro-effects on plant stress responses. Semin Cell Dev Biol, 21(8), 805-811. http://dx.doi.org/10.1016/j.semcdb.2010.04.001

Sunkar, R., \& Zhu, J. K. (2004). Novel and stress-regulated microRNAs and other small RNAs from Arabidopsis. The Plant Cell, 16(8), 2001-2019. http://dx.doi.org/10.1105/tpc.104.022830

Wang, J. W., Czech, B., \& Weigel, D. (2009). miR156-regulated SPL transcription factors define an endogenous $\begin{array}{lllll}\text { flowering pathway in Arabidopsis thaliana. } & \text { 738-749. }\end{array}$ http://dx.doi.org/10.1016/j.cell.2009.06.014

Wang, K., Wang, Z., Li, F., Ye, W., Wang, J., Song, G., ... Yu, S. (2012). The draft genome of a diploid cotton Gossypium raimondii. Nature Genetics, 44, 1098-1103. http://dx.doi.org/10.1038/ng.2371

Wang, L., Gu, X., Xu, D., Wang, W., Wang, H., Zeng, M., ... Cui, X. (2011). miR396-targeted AtGRF transcription factors are required for coordination of cell division and differentiation during leaf development in Arabidopsis. Journal of experimental Botany, 62(2), 761-773. http://dx.doi.org/10.1093/jxb/erq307

Wang, Z. J., Huang, J. Q., Huang, Y. J., Li, Z., \& Zheng, B. S. (2012). Discovery and profiling of novel and conserved microRNAs during flower development in Carya cathayensis via deep sequencing. Planta, 236(2), 613-621. http://dx.doi.org/10.1007/s00425-012-1634-x

Wei, M., Wei ,H., Wu, M., Song, M., Zhang, J., Yu, J., ... Yu S. (2013). Comparative expression profiling of miRNA during anther development in genetic male sterile and wild type cotton. BMC Plant Biology, 13, 66. http //dx.doi.org/10.1186/1471-2229-13-66

Wilson, Z. A., \& Zhang, D. B. (2009). From Arabidopsis to rice: pathways in pollen development. Journal of experimental botany, 60(5), 1479-1492. http://dx.doi.org/10.1093/jxb/erp095

Wu M.F., Tian Q., \& Reed J.W. (2006). Arabidopsis microRNA167 controls patterns of ARF6 and ARF8 expression, and regulates both female and male reproduction. Development, 133(21), 4211-4218. http://dx.doi.org/10.1242/dev.02602

Xie, K., Wu, C., \& Xiong, L. (2006). Genomic organization, differential expression, and interaction of SQUAMOSA promoter-binding-like transcription factors and microRNA156 in rice. Plant physiology, 142(1), 280-293. http://dx.doi.org/10.1104/pp.106.084475

Yang, F., Liang, G., Liu, D., \& Yu, D. (2009). Arabidopsis MiR396 Mediates the Development of Leaves and Flowers in Transgenic Tobacco. Journal of Plant Biology, 52(5), 475-481. http://dx.doi.org/10.1007/s12374-009-9061-7

Yang, J. H., Han, S. J., Yoon, E. K., \& Lee, W. S. (2006). Evidence of an auxin signal pathway, 
microRNA167-ARF8-GH3, and its response to exogenous auxin in cultured rice cells. Nucleic Acids Res, 34(6), 1892-1899. http://dx.doi.org/10.1093/nar/gk1118

Yin, Z., Li, C., Han, X., \& Shen, F. (2008). Identification of conserved microRNAs and their target genes in tomato (Lycopersicon esculentum). Gene, 414(1-2), 60-66. http://dx.doi.org/10.1016/j.gene.2008.02.007

Yin, Z., Li, Y., Yu, J., Liu, Y., Li, C., Han, X., \& Shen, F. (2012). Difference in miRNA expression profiles between two cotton cultivars with distinct salt sensitivity. Mol Biol Rep, 39(4), 4961-4970. http://dx.doi.org/10.1007/s11033-011-1292-2

Zhang, B., Wang, Q., Wang, K., Pan, X., Liu, F., Guo, T., ... Anderson, T. A. (2007). Identification of cotton microRNAs and their targets. Gene 397(1-2), 26-37. http://dx.doi.org/10.1016/j.gene.2007.03.020

Zhang, B. H., Pan, X. P., Cox, S. B., Cobb, G. P., \& Anderson, T. A. (2006). Evidence that miRNAs are different from other RNAs. Cell Mol Life Sci, 63(2), 246-254. http://dx.doi.org/10.1007/s00018-005-5467-7

Supplementary Materials

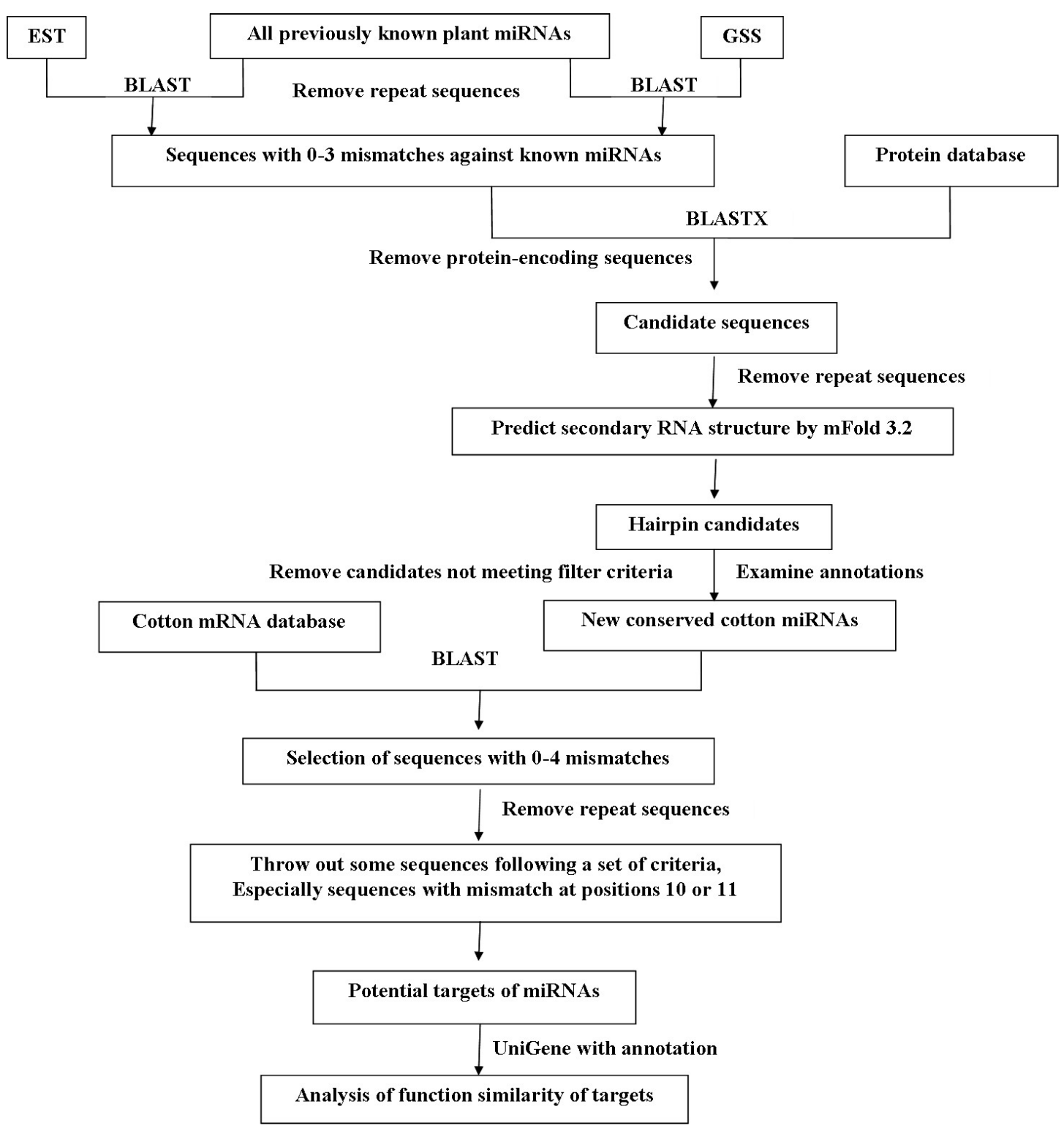

Supplementary Figure 1. Flowchart for the prediction of potential miRNAs and target genes in cotton 


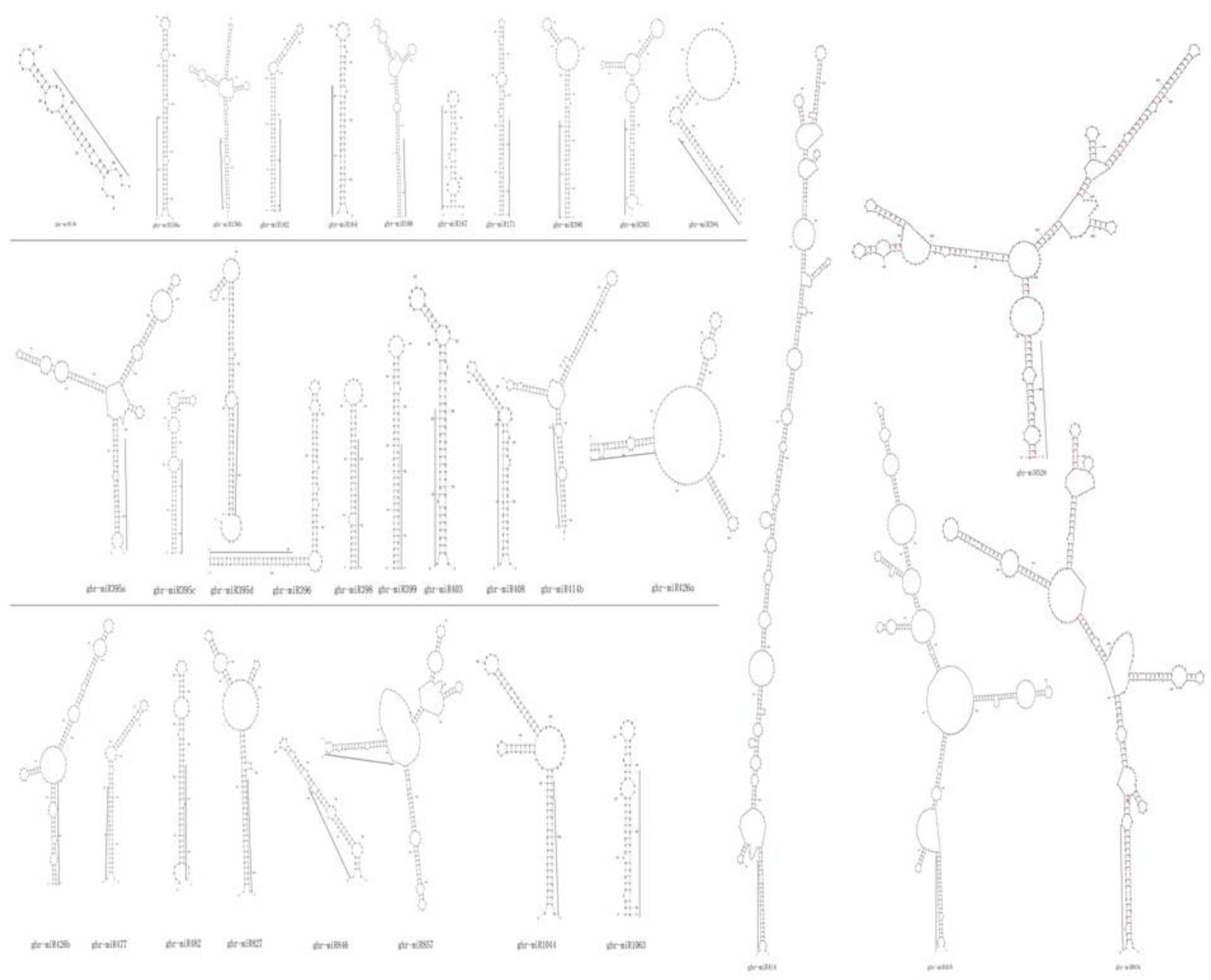

Supplementary Figure 2. Predicted fold-back structures of the cotton miRNA precursors. Sequences of mature miRNAs are underlined 
Supplementary Table 1. Primers used in qRT-PCR

\begin{tabular}{|c|c|c|c|}
\hline Gene & & Primer pairs (5' to $\left.3^{\prime}\right)$ & Predicted amplicon sizes \\
\hline U6 snRNA & F: & GATGACACGCACAAATCGAGAAATG & $105 \mathrm{bp}$ \\
\hline ath-miR157a & F: & GCGGTTGACAGAAGATAGAGAGCA & $104 \mathrm{bp}$ \\
\hline ath-miR167a & F: & GGTGAAGCTGCCAGCATGATCTA & $103 \mathrm{bp}$ \\
\hline ath-miR171a & F: & TGATTGAGCCGCGCCAATATC & $101 \mathrm{bp}$ \\
\hline ath-miR172a & F: & CGAGAATCTTGATGATGCTGCAT & $103 \mathrm{bp}$ \\
\hline ghr-miR156a & F: & GGCCTGACAGAAGAGAGTGAGCAC & $104 \mathrm{bp}$ \\
\hline ghr-miR156b & F: & GCCTGACAGAAGAGAGAGAGCACG & $104 \mathrm{bp}$ \\
\hline ghr-miR157 & F: & CGGATGACAGAAGAGAGAGAGCAC & $104 \mathrm{bp}$ \\
\hline ghr-miR162 & F: & GCTCGATAAACCTCTGCATCCAG & $103 \mathrm{bp}$ \\
\hline ghr-miR164 & F: & AGAAGCAGGGCACGTGCAAA & $100 \mathrm{bp}$ \\
\hline ghr-miR166 & F: & GGACCAGGCTTCATTCCCCA & $100 \mathrm{bp}$ \\
\hline ghr-miR171 & F: & AGATTGAGCCGCGCCAATATC & $101 \mathrm{bp}$ \\
\hline ghr-miR396 & F: & CGTTCCACAGCTTTCTTGAACTG & $103 \mathrm{bp}$ \\
\hline ghr-miR398 & F: & GGTGTGTTCTCAGGTCACCCCTT & $103 \mathrm{bp}$ \\
\hline ghr-miR472 & F: & CTTGCCTACTCCACCCATGCC & $101 \mathrm{bp}$ \\
\hline ghr-miR482 & F: & GCTCTTTCCTACTCСТCССАТАCC & $104 \mathrm{bp}$ \\
\hline ghr-miR834 & F: & TGGTAGCAGCAGTGGTGGTGC & $101 \mathrm{bp}$ \\
\hline vvi-miR166b & $\mathrm{F}:$ & TCGGACCAGGCTTCATTCCTC & $101 \mathrm{bp}$ \\
\hline \multirow[t]{2}{*}{ UBI1 } & F: & TGCTGGGAAACAACTGGAAG & $82 \mathrm{bp}$ \\
\hline & $\mathrm{R}:$ & GAAGACGAAGAACAAGGTGAAG & \\
\hline \multirow[t]{2}{*}{ Ghi.3540 } & F: & GATTCCATCGTTGTCATCCTCGT & $105 \mathrm{bp}$ \\
\hline & $\mathrm{R}:$ & GCAAATTTCTTGTTGGGTCTAGC & \\
\hline \multirow[t]{2}{*}{ Ghi.20857 } & F: & CGCTAATGGAATCGGTGTAAAT & $138 \mathrm{bp}$ \\
\hline & $\mathrm{R}:$ & GAAGCAGACCAAACGGGAAAGT & \\
\hline \multirow[t]{2}{*}{ Ghi.20529 } & F: & GAAGCAAGAACGGTGGTGGG & $172 \mathrm{bp}$ \\
\hline & $\mathrm{R}:$ & GGTTGGTGGTGTAGGAAGCC & \\
\hline \multirow[t]{2}{*}{ Ghi.9257 } & F: & GAACAGATGGGAAGAAATGGAGG & $194 \mathrm{bp}$ \\
\hline & $\mathrm{R}:$ & TGGTTGGTGGTGCCATCAGA & \\
\hline
\end{tabular}

F, forward; R, reverse. 
Supplementary Table 2 . The potential target genes of cotton miRNAs

\begin{tabular}{|c|c|c|c|c|c|}
\hline miRNAs & Target genes & $\begin{array}{l}\text { Reference } \\
\text { protein }\end{array}$ & $\operatorname{Id}(\%)$ & Targeted protein & Target function \\
\hline \multirow[t]{6}{*}{ ghr-miR156b } & Cotton_D_gene_10011417 & & & Transcription factor, SBP-box & Transcription factor \\
\hline & Ghi.13781 & NP_565771.1 & 75.2 & SPL3 & \\
\hline & Ghi.16537 & NP_175723 & 59.9 & SPL4 & \\
\hline & Ghi.18144 & NP_181749 & 46.7 & SPL9 & \\
\hline & Ghi.3540 & NP_001031096 & 49.1 & SPL10 & \\
\hline & Ghi.17877 & NP_193401 & 63.6 & RNA helicase & Metabolism \\
\hline \multirow[t]{3}{*}{ ghr-miR164 } & Cotton_D_gene_10004296 & & & No apical meristem (NAM) protein & Metabolism \\
\hline & Cotton_D_gene_10027143 & & & Spc97/Spc98 & \\
\hline & Ghi.20529 & XP_002321447.1 & 48.8 & NAC domain protein, IPR003441 & Transcription factor \\
\hline ghr-miR166 & Cotton_D_gene_10031827 & & & $\begin{array}{l}\text { homeobox-leucine zipper protein } \\
\text { ATHB-15 }\end{array}$ & Transcription factor \\
\hline \multirow[t]{3}{*}{ ghr-miR167 } & Cotton_D_gene_10000564 & & & xylose isomerase & Metabolism \\
\hline & Ghi.17868 & NP_198518.1 & 61.1 & ARF8 & Transcription factor \\
\hline & Ghi.20503 & NP_174323.1 & 69.9 & ARF6 & \\
\hline \multirow[t]{2}{*}{ ghr-miR171 } & Cotton_D_gene_10000598 & & & Transcription factor GRAS & Transcription factor \\
\hline & Ghi.20857 & NP_191622.1 & 47.7 & scarecrow-like(SCL) protein 22 & \\
\hline ghr-miR393 & Cotton_D_gene_10001907 & & & transport inhibitor response 1 & Metabolism \\
\hline ghr-miR394 & Cotton_D_gene_10011419 & & & F-box domain, Skp2-like & Metabolism \\
\hline ghr-miR396 & Ghi.9257 & NP_001152607.1 & 50 & growth-regulating factor & Transcription factor \\
\hline ghr-miR403 & Ghi.14698 & XP_002332150.1 & 76.4 & Argonaute protein group & Metabolism \\
\hline ghr-miR414 & Cotton_D_gene_10026330 & & & $\begin{array}{l}\text { Late embryogenesis abundant protein, } \\
\text { group } 2\end{array}$ & Metabolism \\
\hline \multirow[t]{2}{*}{ ghr-miR414b } & Cotton_D_gene_10026173 & & & Cytidylyltransferase & Metabolism \\
\hline & Cotton_D_gene_10037360 & & & Nucleosome assembly protein (NAP) & \\
\hline \multirow[t]{2}{*}{ ghr-miR418 } & Ghi.19228 & XP_002984921.1 & 54.7 & MADS-domain transcription factor & Transcription factor \\
\hline & Ghi.13547 & NP_173910.1 & 52 & Protein kinase-like protein & Metabolism \\
\hline \multirow[t]{3}{*}{ ghr-miR426a } & Ghi.19380 & NP_191599.1 & 58.5 & $\begin{array}{l}\text { Mitochondrial transcription termination } \\
\text { factor family protein }\end{array}$ & $\begin{array}{l}\text { Transcription } \\
\text { termination factor }\end{array}$ \\
\hline & Ghi.11224 & NP_180441.1 & 100 & Histone H4 & Metabolism \\
\hline & Ghi.39 & & & H+-pyrophosphatase (VPP) & Metabolism \\
\hline ghr-miR477 & Cotton_D_gene_10021396 & & & Transcription factor GRAS & Metabolism \\
\hline ghr-miR482 & Ghi.2659 & NP_187400.1 & 73.4 & Uncharacterized protein & \\
\hline ghr-miR529 & Ghi.6793 & NP_174462.1 & 80.7 & Acyl-CoA-binding protein & Metabolism \\
\hline ghr-miR834 & Cotton_D_gene_10001209 & & & Homeodomain-like & Metabolism \\
\hline \multirow[t]{2}{*}{ ghr-miR846 } & Cotton_D_gene_10004691 & & & Coproporphyrinogen III oxidase & Metabolism \\
\hline & Ghi.15414 & NP_051066.1 & 86.4 & ATP synthase CF1 beta subunit & \\
\hline ghr-miR1044 & Ghi.21038 & - & - & Unknown protein & \\
\hline ghr-miR1063 & Cotton_D_gene_10023229 & & & Zinc finger, RanBP2-type & Transcription factor \\
\hline
\end{tabular}

Id(\%), the percent identity of sequence alignments; SPL, squamosa-promoter-binding-like protein; ARF, auxin response factor.

\section{Copyrights}

Copyright for this article is retained by the author(s), with first publication rights granted to the journal.

This is an open-access article distributed under the terms and conditions of the Creative Commons Attribution license (http://creativecommons.org/licenses/by/3.0/). 